\title{
Blind Assessment of Image Blur using the Haar Wavelet
}

\author{
Asheer Bachoo \\ Optronic Sensor Systems \\ Defence Peace Safety and Security (DPSS) \\ Council for Scientific and Industrial Research (CSIR) \\ Pretoria, South Africa \\ abachoo@csir.co.za
}

\begin{abstract}
Images and video captured in real world situations generally have distorted digital pixel values. A variety of situations can cause these image degradations: sensor motion, environmental conditions and random noise. A crucial procedure in computer vision is the assessment and quantification of digital image quality. A numerical score for describing image quality is useful for a number of applications, some of which include improving the performance of an image acquisition system and adaptive algorithms. We present an intuitive quality metric for characterizing the amount of blur in an image, through blind image assessment, using the Haar discrete wavelet transform. Thus, the method does not require a reference image or any prior information. The novelty of our method lies in processing the image derivative using the discrete wavelet transform rather than directly processing image intensity values as is traditionally done. We present late breaking results and analysis for a small set of data. The proposed method shows promise for a large number of avenues such as realtime blur level assessment and image depth of focus estimation.
\end{abstract}

\section{Categories and Subject Descriptors}

I.4.7 [Image Processing and Computer Vision]: Feature Measurement-Invariants

\section{General Terms}

Algorithms

\section{Keywords}

no-reference image quality assessment, blind image quality assessment, image blur characterization

\section{INTRODUCTION}

Image degradation is frequently experienced when using imaging technologies in the real world. Several factors present during the acquisition process are largely uncontrolled and manifest in images as blur, noise or low contrast. An important step towards image enhancement and

Permission to make digital or hard copies of all or part of this work for personal or classroom use is granted without fee provided that copies are not made or distributed for profit or commercial advantage and that copies bear this notice and the full citation on the first page. To copy otherwise, to republish, to post on servers or to redistribute to lists, requires prior specific permission and/or a fee.

SAICSIT' 10, October 11-13, Bela Bela, South Africa

Copyright 2010 ACM 978-1-60558-950-3/10/10 ...\$10.00. scene understanding is that of image quality assessment - what is the type and level of degradation in an image? This question is not a trivial one. The assessment of image quality is useful for a number of reasons: i) quantifying the performance of an acquisition or processing system, ii) providing a numerical reference for algorithm or system parameter selection and iii) scene understanding.

Three approaches are present in the literature for image quality assessment. The most common method uses a reference image to estimate description metrics. This is known as full-reference image quality assessment. A second approach is that of reduced-reference image quality assessment which computes a metric using partial knowledge of the reference image. In most real world situations a reference image or partial information is unavailable for image grading. Thus, it is required to compute an image quality metric without the use of a reference image. This is called blind (no-reference) image quality assessment and is the subject of this paper. Avcibass et al. [1] provide a comprehensive statistical evaluation of image quality metrics using full-reference.

The definition of image quality is highly subjective and dependent on a cognitive process that is currently not easily modelled in computer vision. This cognitive process is a product of years of observation, memory retention and the emotional and mental development of the observer. The human perception of "good" or "bad" images, in conjunction with the cognitive process, is also determined by the task at hand. As such image quality assessment at an objective level is dependent on the application requirements and the criteria for assessment. For example, it may be required to measure the focus of an optical system. In this case, a "good" image is one that is in focus or has a great depth of field. Thus, the image quality metric will be objective and consistent for different images and it will be defined by the user.

In this short paper, we focus on describing one component of no-reference image quality assessment - quantifying the level of blur (or defocus) in an image. The proposed algorithm forms part of a method (in our current research) to perform general image quality assessment using the discrete wavelet transform. Within this framework image blur, noise and information content are characterized. Thus, using a single transform, several image characteristics can be estimated at a low computational cost making real-time assessment and correction of distorted images possible. The ability to quantify and interpret several factors in image quality, without a reference image, also provides a good understanding of the environment and the acquisition hardware. The discrete wavelet transform also provides multiscale spatial and frequency decomposition. The frequencies can be resolved in space 


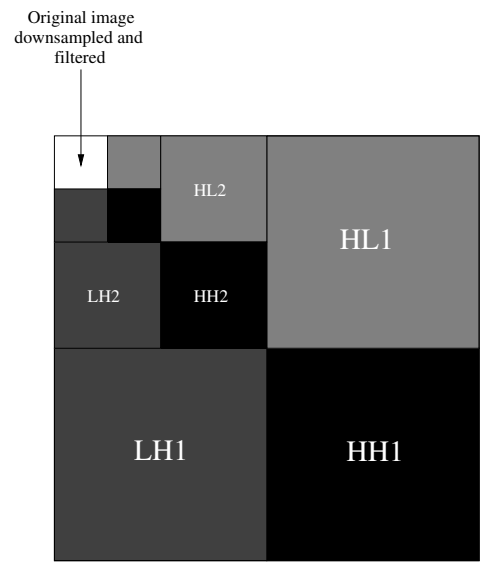

Figure 1: 2-Dimensional Discrete Wavelet Transform Decomposition Process

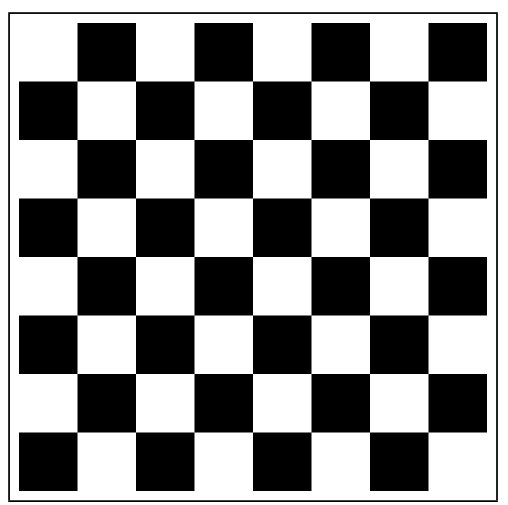

Figure 2: Checker Test Pattern

and this is very useful for locating particular features of interest in an image. For this reason, it is preferred over other methods such as the Fourier transform.

We first provide general background on the field of noreference blur assessment in Section 2. In Section 3, the method used for computing the blur metric is proposed. Thereafter, the experimental analysis, discussion and conclusions are presented.

\section{BACKGROUND}

Cohen and Yitzhaky [3] present a metric for assessing blur and noise impacts in images. In the frequency domain, blur impacts are measured using an average image power spectrum model multiplied by a weighting function. The resulting weighted spatial frequency spectrum shows a decreasing slope in the presence of blur.

Nill and Bouzas presented a quality metric computed using the image power spectrum with an incorporated human visual system model [9]. A Wiener filter is used to model system noise. The metric can distinguish between blur and noise only when separately added to the measured image. It is assumed that the original image power spectrum is independent of content.

Marziliano et al. [8] propose a no-reference perceptual blur metric. The method first locates vertical edges and then it finds the start and end of each vertical edge by looking at the closest local extrema locations (in the horizontal directions). The width for a particular edge location is computed as the difference between the start and end positions of the edge. The global blur metric is the average of all edge widths in the image. Robustness of this method is dependent on having quality step edges in the scene.

Gabarda and Cristobel propose computing the Rényi entropy of a spatial/spatial-frequency distribution [4]. An oriented pseudo-Wigner distribution is used for this purpose. The variance of the entropy is computed as a function of directionality and used as a quality indicator. In their experiments, the metric is able to correctly grade a set of images according to the human perception of "good" and "bad" (when blurred or corrupted by random noise). Although this is useful, the computed score of a solitary image will not provide the user with an understanding of the actual image quality.

Li proposes several metrics for blind image assessment [6]. These metrics are edge sharpness level, random noise level and structural noise level. However, the results presented, though promising, are not conclusive since only a single test image is shown. The next section explains the method used in computing the proposed image quality metric for blur.

\section{PROPOSED METHOD}

The proposed image blur metric is estimated through an application of the Haar discrete wavelet [5] transform on the image derivative and not on the image intensities as is traditionally done. The following assumptions are made when characterizing the image blur:

1. Within the scope of this paper, the input images are deemed to be noise free. This is a normal assumption since the test data set consists of natural scenes with a low level of noise. Noise impacts will be assessed in future work using the robust noise estimation method of Chang et al. [2].

2. It is assumed that the image defocus can be estimated through the processing of edge information. The smoothing of an edge indicates blur while a strong step edge indicates sharpness. Thus, the input image should have a sufficient number of step edges. The Haar wavelet transform is able to process extremely smooth edges and determine the scale at which they become step edges. Our method is superior to that of Marziliano et al. [8] because we analyse edge information at multiple scales and noise is filtered out across scales by the wavelet transform.

The discrete wavelet transform is applied to a 2-dimensional (2D) digital image $f(x, y)$ by filtering across the rows and then the columns of the result. This is followed by downsampling by a factor 2 to achieve the effect of scaling. The transform decomposes the image into Low-Low (LL), Low-High (LH), High-Low (HL) and High-High (HH) frequency components at multiple scales. Figure 1 shows the $2 \mathrm{D}$ spatial decomposition at several scales.

When processing the image derivative, wavelet coefficients with significantly large magnitudes at a particular scale will represent edge points in the image. The averaging and differencing operation of the Haar wavelet highlights the scale at which an edge is most visible i.e. the scale at which it becomes a step edge. If the energy is computed for each scale, and normalized by the sum total energy, it can be seen that the scale with the largest energy intuitively describes the dominant level of blur, or edge width, in the image. The energy at each level represents the difference in energy between the current and previous scale i.e. it is bandpass filtered. 

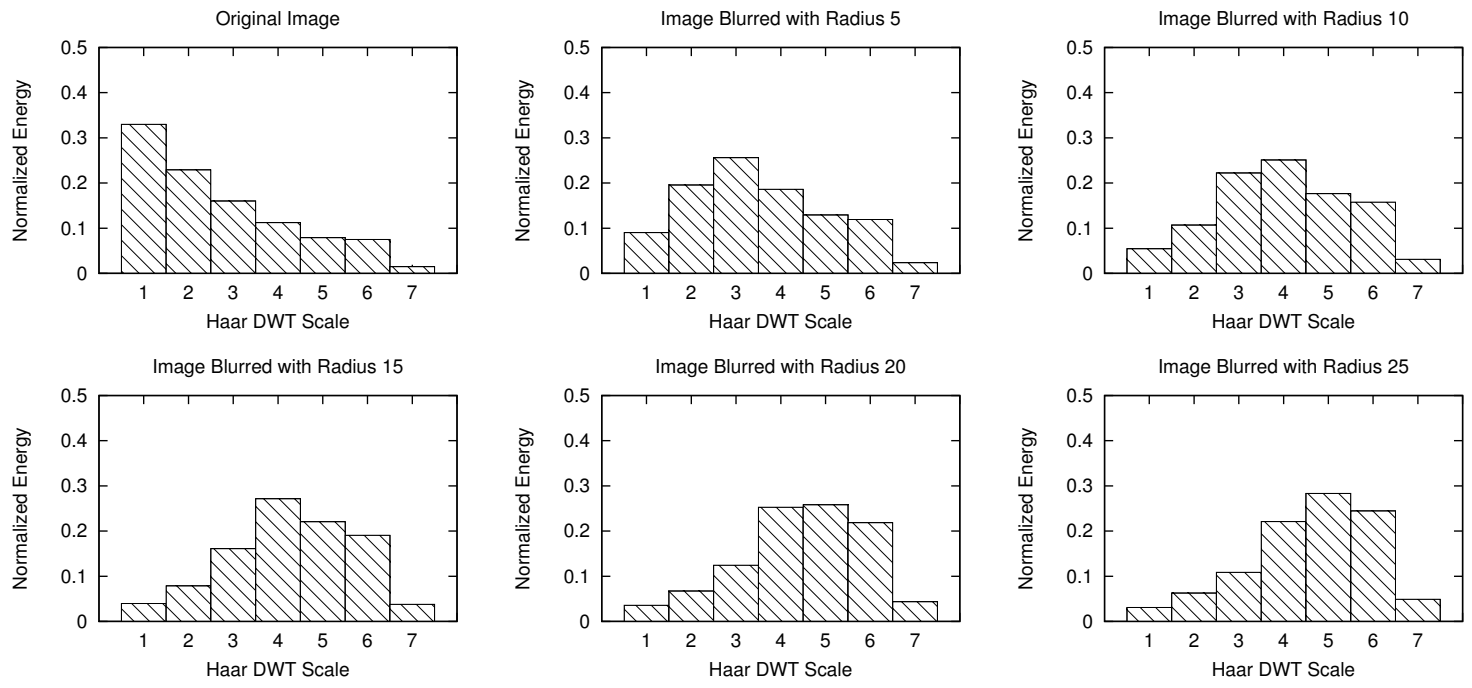

Figure 3: Checkerboard Energy Change for Different Blur Levels

We first compute the two first derivatives of the image, $d_{x}(x, y)$ and $d_{y}(x, y)$, using the 2 well known vertical and horizontal $3 \times 3$ Sobel operators for edge detection [10]. A number of methods are available for computing the image derivative [7] and this is left to the interested reader for exploration. Thereafter, we transform $d_{x}$ and $d_{y}$ using the Haar wavelet. For each scale $s$, the energies for $H L$ and $L H$ in both $d_{x}$ and $d_{y}$ are summed. The total normalized energy at scale $s$ is represented by the following equation:

$$
e_{s}=\frac{\sum c(x, y)^{2}}{E}
$$

where $E$ is the total energy computed as the sum of all $\sum c(x, y)^{2}$ across all scales. In the above equation $\sum c(x, y)^{2}$ is the sum of all wavelet coefficient squares at scale $s$. These coefficients exist in either $d_{x}$ or $d_{y}$ and are either from components $H L$ or $L H$ (and not $L L$ or $H H$ ). More details regarding the Haar wavelet transform and energy functions can be found in standard texts [5].

To demonstrate the validity of the method, the wavelet energies were computed for a test pattern of size $512 \times 512$ pixels (the checker board shown in Figure 2). The normalized energies for various blur levels are shown in Figure 3. The checker board image was blurred using Gaussian smoothing filters (with the radii in pixels as reported in the Figures). As the level of blur increases, the peak in the energies occurs at a higher scale i.e. the peak in the graphs shifts to the right. This is a general trend observed for all the test images. An important point to mention is that maximizing the energy at the first or second scale, by varying a camera's lens and aperture settings, will produce the sharpest possible image for a scene if step edges are present in the scene.

The image blur quality metric is then computed as follows:

$$
b=\sum_{s=1}^{S} 2^{s-1} e_{s}
$$

where $S$ is the total number of scales used in the wavelet decomposition process. The term $2^{s-1}$ can best be described as the size of a feature for a pixel at scale $s$. The above equation computes the blur score for an image as a sum of weighted feature sizes.

\section{EXPERIMENTAL ANALYSIS AND DIS- CUSSION}

The experimental data set consisted of 20 natural grey scale (8-bit) images of size $512 \times 512$ pixels. Some of the images are shown in Figure 4 . These images are noise free and most of the foreground and background are in focus - this is important for consistency and integrity of the experimental results. For the sake of consistency, it is assumed that the images have the greatest sharpness when in their original state. This was verified through a visual inspection by the author. However, it must be mentioned that in real world scenarios an acquired image may not tend to be completely sharp due to varying lens and aperture configurations that reduce the depth of focus. This, and the effects of additive noise, will be assessed in future work.

If a set of test images has different resolutions, the images should be scaled appropriately to achieve a standard edge scale. For example, if a perfect step edge has a width of 2 pixels, this should be ensured for all input images in order to make the results consistent and comparable. Thus, it is preferred that the edge scale be consistent. This can be achieved quite easily by ensuring that the test images are acquired using the same camera system and settings or by visual inspection of the data. For comparison to the results in this paper, the data set can be requested from the author. The results presented in this paper are merely for a proof of concept.

The experiments conducted in this paper examine the estimated blur metric and how it changes as the blur level in the image is increased. The effects of blur are induced using the circular symmetric 2D Gaussian smoothing function. For this study, we used five levels of blur. These levels, in number of pixels for the blur radius, were in the set $\{0,5,10,15,20,25\}$ where zero refers to the original image.

Given the original images and their blurred variations, the image blur score was computed for each image using Equation 2 and 7 wavelet scales. The mean and standard deviation was then computed for each blur level over 20 images. Figure 5 (showing error bars) and Table 1 summarizes these results. It is quite apparent that for each blur level up to radius 15 pixels, the means are signif- 

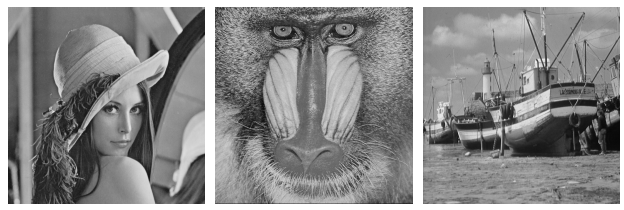

Figure 4: Some Test Images for the Blur Assessment

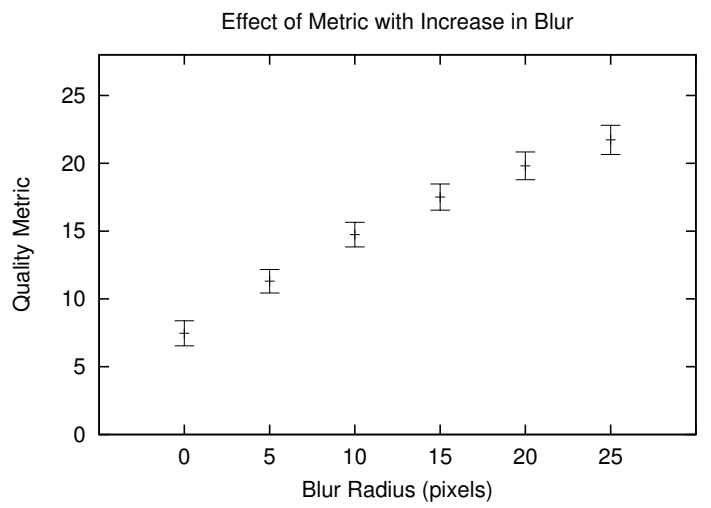

Figure 5: Quality Metric over Different Scales

Table 1: Results for 20 Images

\begin{tabular}{|l|c|c|}
\hline $\begin{array}{l}\text { Blur Level } \\
\text { (pixels) }\end{array}$ & Mean & Std. Dev. \\
\hline Original & 7.46 & 0.92 \\
\hline 5 & 11.30 & 0.86 \\
\hline 10 & 14.74 & 0.91 \\
\hline 15 & 17.50 & 0.96 \\
\hline 20 & 19.80 & 1.02 \\
\hline 25 & 21.72 & 1.07 \\
\hline
\end{tabular}

icantly different with a fairly low standard deviation of less than 1.0. For consecutive blur levels, there is minimal overlap between the blur scores. Thus, one can perform a coarse level blur estimation by computing the blur score and looking at Table 1. For blur levels of 20 and 25 pixel radii, there appears to be a significant overlap between the ranges of the estimated scores. It is quite clear that the inter-mean distance decreases as blur level increases. From these observations, it can be concluded that the current blur score is useful for Gaussian blur levels up to radius 15 pixels. The variations in quality scores for each blur level can be attributed to subtle differences in depth of focus and small amounts of noise in the images that affects the wavelet energies.

In terms of computer execution, the average computation time for the metric is approximately 0.025 seconds (40 frames-per-second). This includes a large number of overheads such as image input, data conversion, memory allocation and copying data to memory. Thus, real-time blur assessment and quantification is highly possible when using the proposed method.

The current work is very promising for blur estimation in an image. Two important components will be undertaken in our future work: i) analysis of the blur metric across a larger range of blur degradations and ii) measurement of the error when predicting the blur level in an image using training data of natural scenes. The monotonic behaviour of the blur quality metric will also be assessed further through pattern classification. The implication is that a function can be used to estimate the level of blur in an image when provided with the blur score. Using basic curve fitting through the means in Table 1, we achieved 0.81 and 0.77 root-mean-square error for a log and straight line function respectively. Our future work will also use the technique of Chang et al. [2], called BayesShrink, for quantifying additive white noise in an image. In terms of image processing, it will be worth examining ways to select useful edges for processing rather than processing all edges in the image. The local analysis of image blur will also provide a more accurate assessment and a coarse level depth of focus description if scene and camera information are incorporated.

\section{CONCLUSION}

The proposed method shows a fair consistency for quantifying image blur in natural scenes. The quality metric is descriptive and can be interpreted at a coarse level by a human operator using the tabled results. The method is effective for Gaussian blur up to radius 15 pixels when using the reported dataset. If the blur level is greater than 15 pixels in radius, the metric is still useful for knowing the extent of blurring. However, resolving the amount of blur will not be possible. The technique used to compute the image blur score is well established in the literature and, thus, provides a firm grounding for the proposed method. Execution times for computing the blur score are in the order of 40 frames-per-second and this is very useful for real time image assessment.

\section{REFERENCES}

[1] I. Avcibạs, B. Sankur, and K. Sayood. Statistical evaluation of image quality measures. Journal of Electronic Imaging, 11:206-223, 2002.

[2] S. Chang, B. Yu, and M. Vetterli. Adaptive wavelet thresholding for image denoising and compression. IEEE Transactions on Image Processing, 9(9):1532-1546, 2000.

[3] E. Cohen and Y. Yitzhaky. No-reference assessment of blur and noise impacts on image quality. Signal, Image and Video Processing, 2009.

[4] S. Gabarda and G. Cristobel. Blind image quality assessment through anisotropy. J. Opt. Soc. Am. A, 24(12), 2007.

[5] R. Gonzalez and R. Woods. Digital image processing. Addison-Wesley Publishing Company, 2002.

[6] X. Li. Blind image quality assessment. In IEEE ICIP, 2002.

[7] M. Sonka, V. Hlavac and R. Boyle. Image Processing: Analysis and Machine Vison. PWS Publishing Company, 1999.

[8] P. Marziliano, F. Dufaux, S. Winkler, and T. Ebrahimi. A no-reference perceptual blur metric. In International Conference on Image Processing, volume 3, pages 57-60, Rochester, NY, Sept. 22-25, 2002.

[9] N. Nill and B. Bouzas. Objective image quality measure derived from digital image power spectra. Optical Engineering, 31(4):813-825, 1992.

[10] I. Sobel. Camera Models and Machine Perception. $\mathrm{PhD}$ thesis, Stanford University, Palo Alto, California, 1970. 